\title{
A Matter of Urgency: Reducing Clinical Text Message Interruptions During Educational Sessions
}

\author{
Arielle Mendel, $\mathrm{MD}^{1,2}$, Anthony Lott, MD11, Lisha Lo, $\mathrm{MPH}^{3}$, Robert Wu, MD, MSc ${ }^{1,4 *}$
}

'Division of General Internal Medicine, Department of Medicine, University Health Network, Toronto, Ontario, Canada; ${ }^{2}$ Division of Rheumatology, Department of Medicine, McGill University, Montreal, Quebec; Canada ${ }^{3}$ Centre for Quality Improvement and Patient Safety, Faculty of Medicine, University of Toronto, Toronto, Ontario, Canada; ${ }^{4}$ Department of Medicine, University of Toronto, Toronto, Ontario, Canada.

BACKGROUND: Text messaging is increasingly replacing paging as a tool to reach physicians on medical wards. However, this phenomenon has resulted in high volumes of nonurgent messages that can disrupt the learning climate.

OBJECTIVE: Our objective was to reduce nonurgent educational interruptions to residents on general internal medicine.

DESIGN, SETTING, PARTICIPANTS: This was a quality improvement project conducted at an academic hospital network. Measurements and interventions took place on eight general internal medicine inpatient teaching teams.

INTERVENTION: Interventions included (1) refining the clinical communication process in collaboration with nursing leadership; (2) disseminating guidelines with posters at nursing stations; (3) introducing a noninterrupting option for message senders; (4) audit and feedback of messages; (5) adding an alert for message senders advising if a message would interrupt educational sessions; and (6) training and support to nurses and residents.
MEASUREMENTS: Interruptions (text messages, phone calls, emails) received by institution-supplied team smartphones were tracked during educational hours using statistical process control charts. A one-month record of text message content was analyzed for urgency at baseline and following the interventions.

RESULTS: The interruption frequency decreased from a mean of $0.92(95 \% \mathrm{Cl}, 0.88$ to 0.97$)$ to $0.59(95 \% \mathrm{Cl}$, 0.51 to 0.67$)$ messages per team per educational hour from January 2014 to December 2016. The proportion of nonurgent educational interruptions decreased from $223 / 273(82 \%)$ messages over one month to $123 / 182$ $(68 \% ; P<.01)$.

CONCLUSIONS: Creation of communication guidelines and modification of text message interface with feedback from end-users were associated with a reduction in nonurgent educational interruptions. Continuous audit and feedback may be necessary to minimize nonurgent messages that disrupt educational sessions. Journal of Hospital Medicine 2018;13:616-622. Published online first April 25, 2018. () 2018 Society of Hospital Medicine

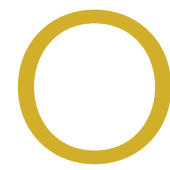

$\mathrm{n}$ general medical wards, effective interprofessional communication is essential for high-quality patient care. Hospitals increasingly adopt secure text-messaging systems for healthcare team members to communicate with physicians in lieu of paging. ${ }^{1-3}$ Text messages facilitate bidirectional communication ${ }^{4,5}$ and increase perceived efficiency ${ }^{6-8}$ and are thus preferred over paging by nurses and trainees. However, this novel technology unintentionally causes high volumes of interruptions. ${ }^{9,10} \mathrm{Com}$ pared to paging, sending text messages and calling smart-

\footnotetext{
*Address for correspondence: Arielle Mendel, MD, Division of General Internal Medicine, Toronto General Hospital, 200 Elizabeth St, Toronto, ON M5G 2C4. Telephone: 416-910-1055; Email: ariellemendel@gmail.com

Additional Supporting Information may be found in the online version of this article.
}

Received: October 31, 2017; Revised: January 11, 2018;

Accepted: January 26, 2018

๑ 2018 Society of Hospital Medicine DOI 10.12788/jhm.2959 phones are more convenient and encourage communication of issues in real time, regardless of urgency. ${ }^{11}$ Interrupting messages are often perceived as nonurgent by physicians..$^{6,12}$ In particular, $73 \%-93 \%$ of pages or messages sent to physicians are found to be nonurgent. . $^{3-17}$

Pages, text messages, or calls not only interrupt day-to-day tasks on the ward $6,7,10,11,17,18$ but also educational sessions, ${ }^{18-21}$ which are essential to the clinical teaching unit (CTU). Interruptions reduce learning and retention ${ }^{22}$ and are disruptive to the medical learning climate. ${ }^{18-20,23}$

Internal medicine CTUs at our large urban academic hospital network utilize a smartphone-based text messaging tool for interdisciplinary communication. Nonurgent interruptions are frequent during educational seminars, which occur at our institution between 8 AM and 9 AM and 12 PM and 1 PM on weekdays. ${ }^{10,11,19}$ In a preliminary analysis at one hospital site, an average of three text messages (range 1-11), two calls (range $0-8$ ), and three emails (range 0-13) interrupted each educational session. Physicians and nurses can disagree on the urgency of messages or calls for the purposes of patient care and workflow. ${ }^{6,11,12,24}$ Nurses have expressed a desire for guidance 

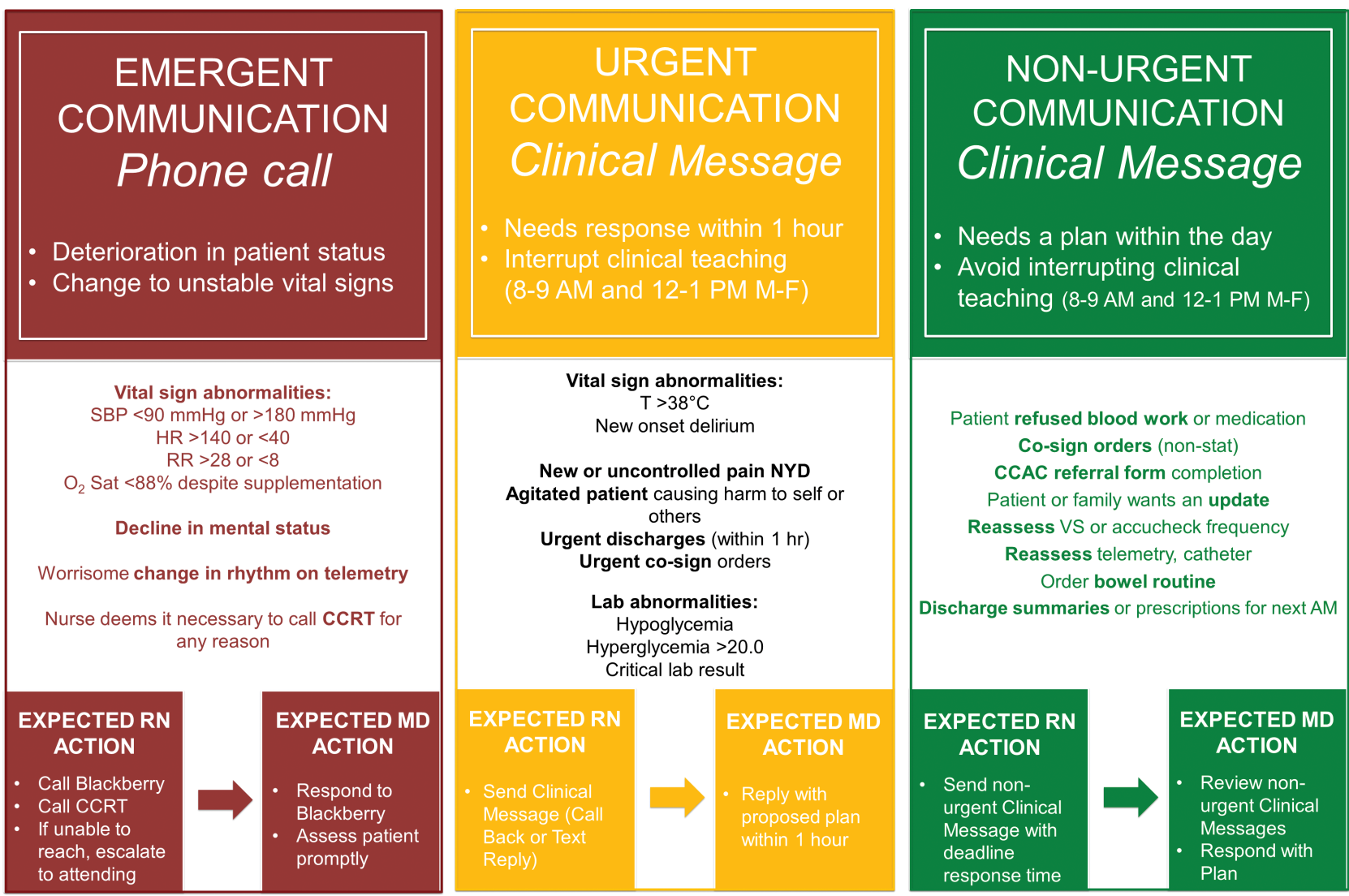

FIG 1. Example of a poster displaying the recommended clinical communication process, developed through interdisciplinary consensus.

regarding what constitutes an urgent clinical communication. ${ }^{6}$

This project aimed to reduce nonurgent text message interruptions during educational rounds. We hypothesized that improved decision support around clinical prioritization and reminders about educational hours could reduce unnecessary interruptions.

\section{METHODS}

This study was approved by the institution's Research Ethics Board and conducted across eight general medical CTU teams at an academic hospital network (Sites 1 and 2). Each CTU team provides 24-hour coverage of approximately 20-28 patients. The most responsible resident from each team carries an institution-provided smartphone, which receives secure texts, phone calls, and emails from nurses, social workers, physiotherapists, speech language pathologists, dieticians, pharmacists, and other physicians. Close collaboration with the platform developer permitted changes to be made to the system when needed. Prior to our interventions, a nurse could send a text message as either an "immediate interrupt" or a "delayed interrupt" message. Messages sent via the "delayed interrupt" option would be added to a queue and would eventually lead to an interrupting message if not replied to after a defined period. Direct phone calls were reserved for especially urgent or emergent communications.
Meetings were held with physicians and nursing managers at Site 1 (August 2014) and Site 2 (January 2015) to establish consensus on the communication process and determine clinical scenarios, regardless of time of day, that warrant a phone call, an "immediate interrupt" text, or a "delayed interrupt" text. In March 2015, resident feedback led to the addition of a third option to the sender interface. This option allowed messages to be sent as "For Your Information (FYI)" only, which would not lead to an interruption. "FYI" messages (for example, to notify that an ambulance had been booked for a patient), were instead placed in an electronic message board that could be viewed by the resident through the application. This change relied upon interdisciplinary trust and a commitment from residents to ensure that "FYI" messages were reviewed regularly.

Communication guidelines were transformed into poster format and displayed as a reference at nursing stations in July 2015 (Site 2) and February 2016 (Site 1; Figure 1). Nurse managers audited messages from nurses and provided feedback. In March 2016, a focused intervention was piloted across both sites to specifically limit nonurgent text messages during educational hours. First, educational hours were emphasized within the interface to make senders aware of their potential for interruption. In June 2016, the interface was further modified. Once the message application was opened during a defined educational time, an imbedded notification advised the 


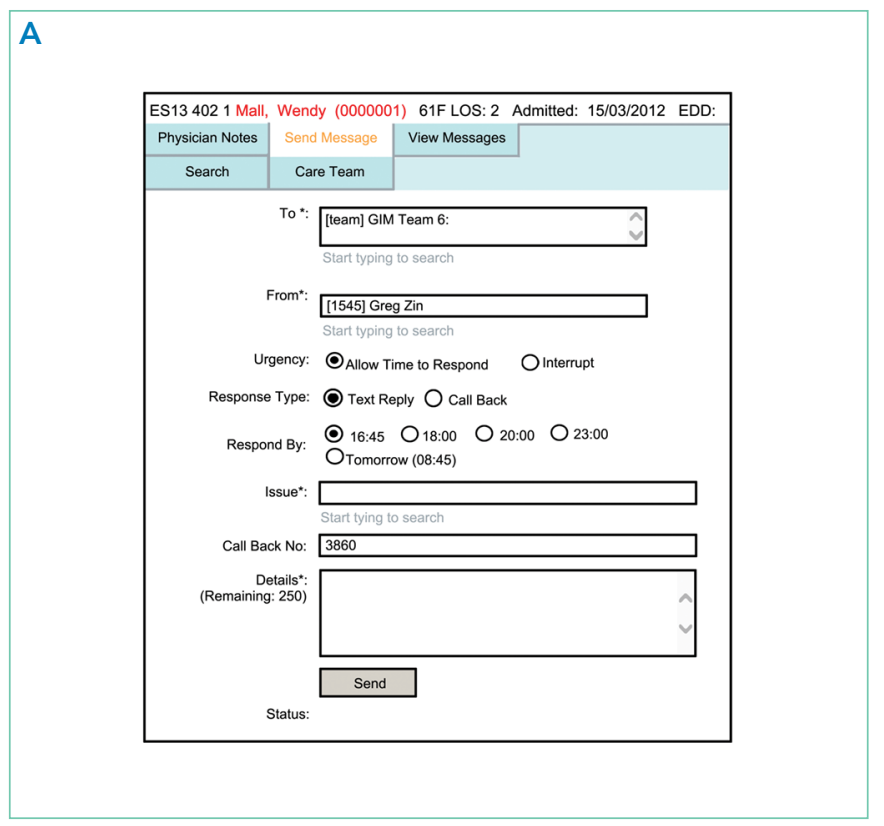

B
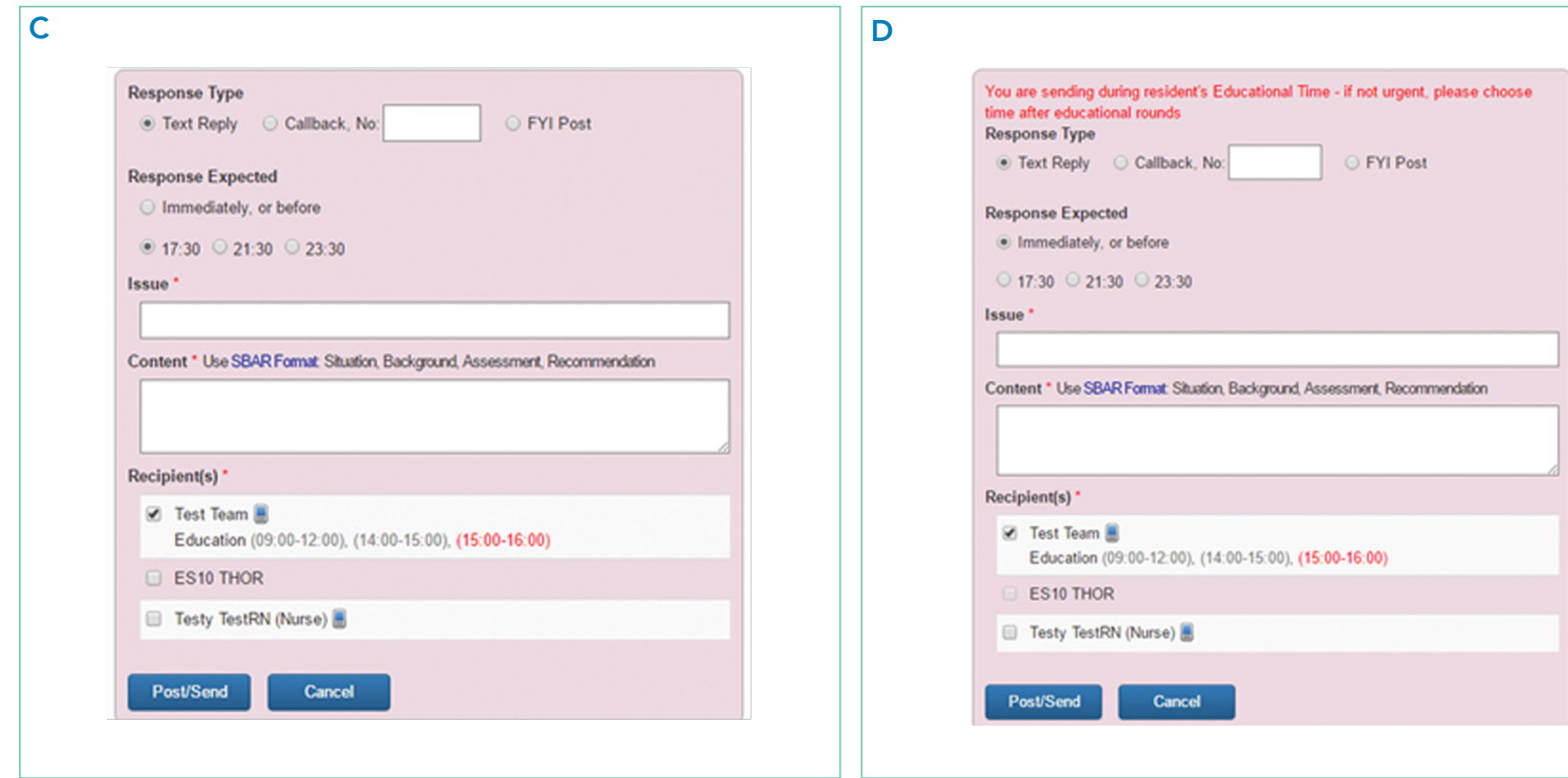

FIG 2. (A) Original text message interface. (B) March 2015: "FYI Post" option created. (C) March 2016: educational times defined in message interface. (D) June 2016: alert in message interface regarding educational hours.

sender to reevaluate the urgency of the communication and if appropriate, to delay sending the message until educational rounds were over or send an "FYI" message. This "alert" did not impede senders from sending a message through the system at any time (Figure 2A-D illustrates the evolution of the message interface).

Text interruptions (January 2014 to December 2016), phone calls (April 2015-December 2016), and emails (October 2014 to December 2016) received by team smartphones during educational hours were tracked. Total text messages sent over a 24hour period and the type of message ("immediate interrupt," "delayed interrupt," and "FYI") were also monitored. Calls were encouraged only in the case of emergent patient care matters, and monitoring calls would thus help identify whether senders bypass the message system due to deterioration in patient status or confusion surrounding the new message interface. Emails sent to team smartphones came from a variety of sources, including hospital administration, physicians, and patient flow coordinators who are not involved in direct patient care. Emails served as a "negative control" because of the predicted random variability in the email interruption frequency. Additional balancing measures included tracking Critical Care Outreach Team consultations and "Code Blue" (cardiac arrest) announcements over the same period to ensure that limiting educational interruptions did not result in increased deterioration of patient status. 


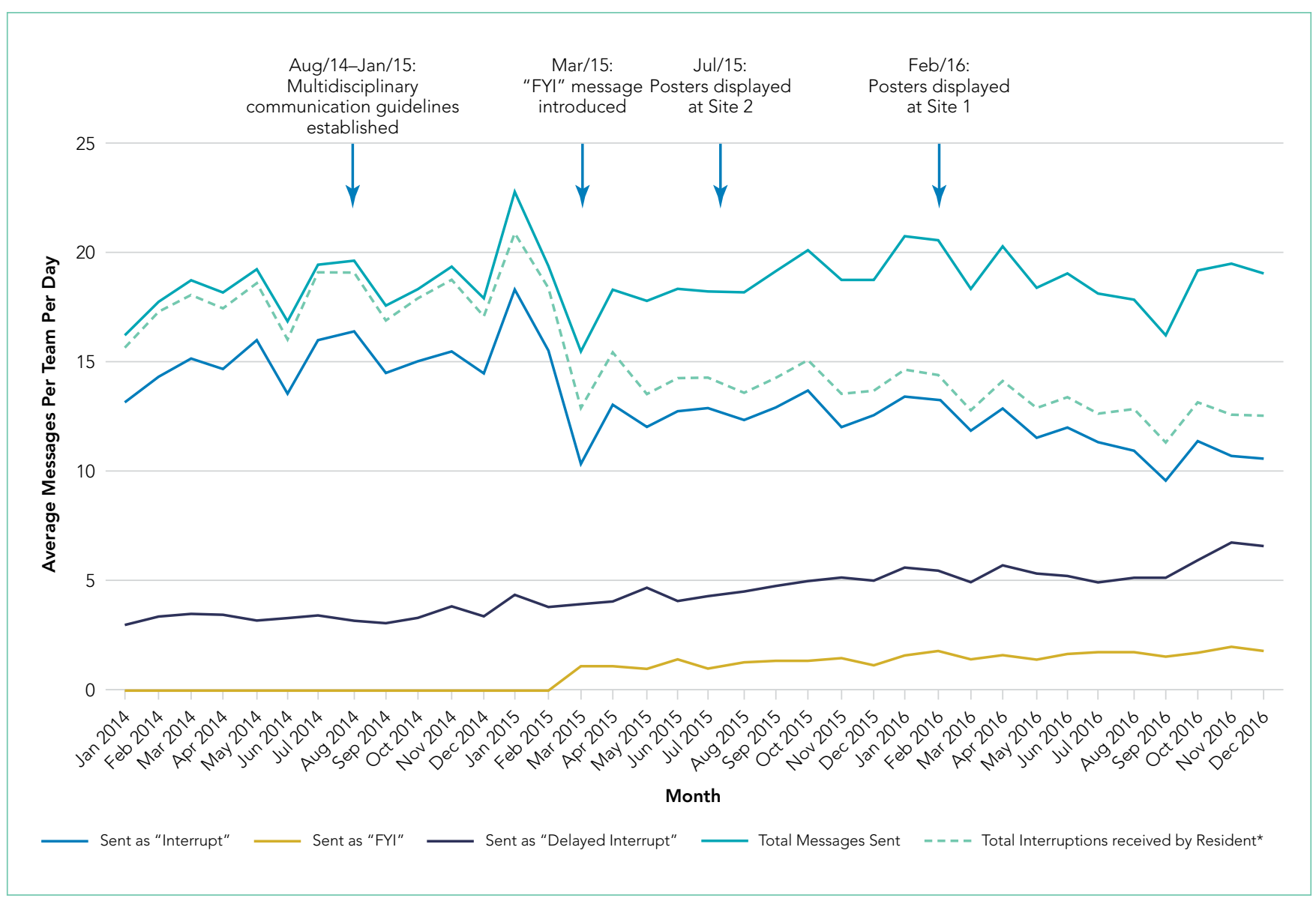

FIG 3. Total text messages sent to team smartphones according to message type, January 2014-December 2016.

*comprises messages sent as "interrupt" plus a portion of those sent as "delayed interrupt" that were not responded to by the time limit, triggering an interruption

Statistical process control charts (u charts) assessed the frequency of each type of educational interruption (text, call, or email) per team on a monthly basis. The total educational interruptions per month were divided by the number of educational hours per month to account for variation in educational hours each month (for example, during holidays when educational rounds do not take place). If call logs or email data were unavailable for individual teams or time periods, then the denominator was adjusted to reflect the number of teams and educational hours in the sample for that month.

Two four-week samples of interrupting text messages received by the eight teams during educational hours were deidentified, analyzed, and compared in terms of content and urgency. A preintervention sample (November 17 to December 14, 2014) was compared to a postintervention sample (November 14 to December 11, 2016). Messages from the 2014 and 2016 samples were randomized, deidentified for date and time, and analyzed for urgency by three independent adjudicators (two senior residents and one staff physician) to avoid biasing the postintervention analysis toward improvement. Messages were classified as "urgent" if the adjudicator felt a response or action was required within one hour. Messages not meeting these criteria were classified as "nonurgent" or "indeterminate" if the urgency of the message could not be assessed because it required further con- text. Fleiss kappa statistic evaluated agreement among adjudicators. Individual urgency designations were compared for each message, and discrepant rankings were addressed through repeated joint assessments. Disagreements were resolved through discussion and comparison against communication guidelines. In addition, messages reporting a "critical lab," requiring physician notification as per institutional policy, were reclassified as "urgent." The proportion of "nonurgent" messages sent during educational hours was compared between baseline and post-intervention periods using the Chi-square test.

"FYI" messages sent from November 14 to December 11, 2016 were audited using the same adjudication process to determine if " $\mathrm{FYI}$ " designations were appropriate and did not contain urgent patient care communications.

\section{RESULTS}

Total text messages sent to team smartphones, the type of message the sender intended ("immediate interrupt," "delayed interrupt," or "FY"), and total text interruptions received by the resident over the study period are illustrated in Figure 3. The introduction of the "FY" message in March 2015 was associated with reduced text message interruptions, from a mean of $18.0(95 \% \mathrm{Cl}, 17.2$ to 18.8$)$ interrupting messages per team per day to $14.1(95 \% \mathrm{Cl}, 13.6$ to 14.5$)$ in March 2015 and 


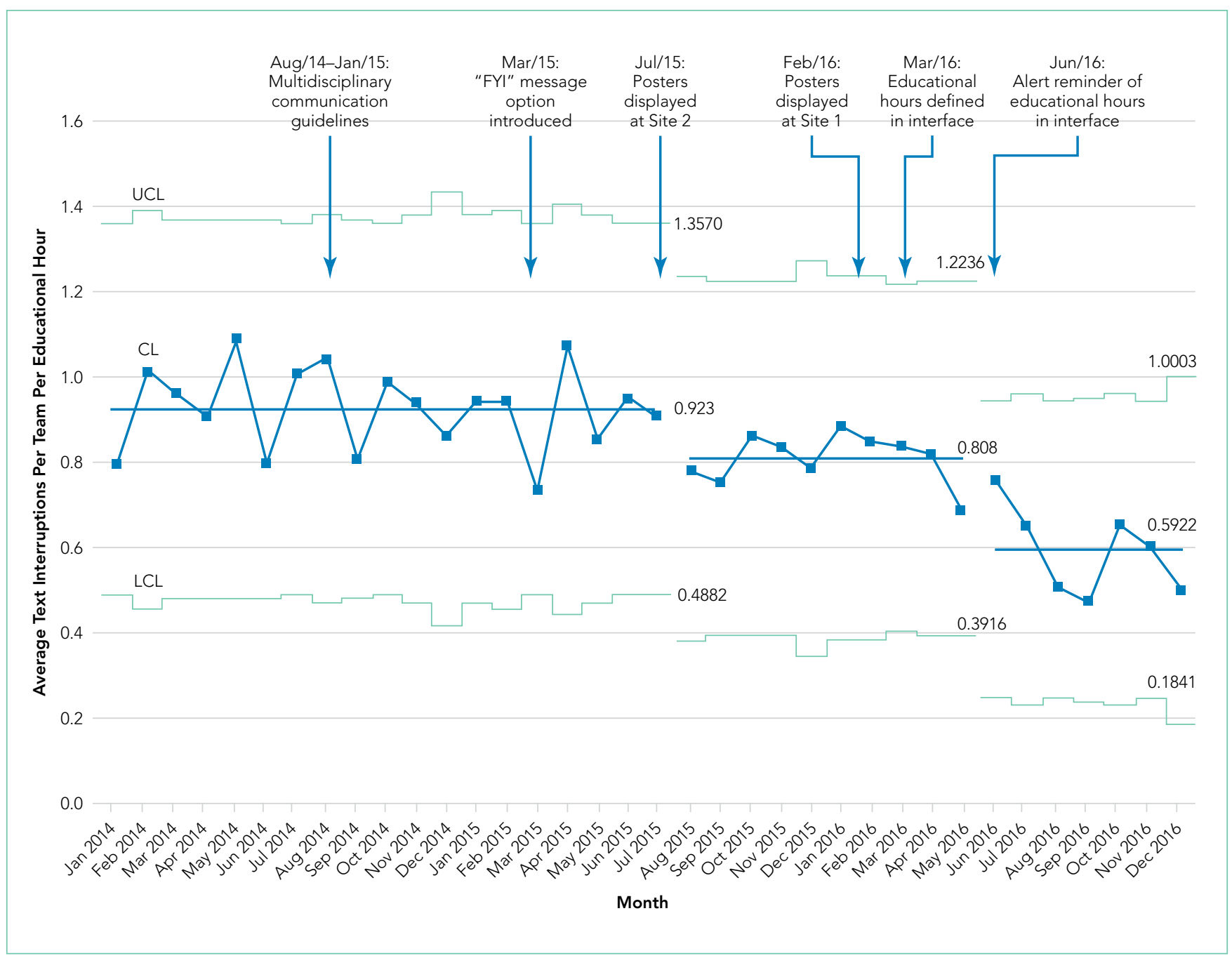

FIG 4. U Chart: text message interruptions per team per educational hour, January 2014-December 2016

12.7 (95\% Cl, 12.2 to 13.2) after May 2016 (Supplemental Figure 1). The numbers of "delayed interrupt" and "FYI" messages increased over time.

Analysis of text interruptions during educational hours indicated three distinct phases (Figure 4). A mean of $0.92(95 \%$ $\mathrm{Cl} 0.88$ to 0.97$)$ text interruptions per team per educational hour was found during the first phase (January 2014 to July 2015). The message frequency decreased to a mean of 0.81 (95\% Cl, 0.77 to 0.84 ) messages per team per educational hour starting August 2015, following the implementation of the "FYI" message option for senders (March 2015) and dissemination of communication guidelines (July 2015). Finally, a further reduction to a mean of $0.59(95 \% \mathrm{Cl}, 0.51$ to 0.67$)$ messages per team per educational hour began in June 2016 after the creation of the alert message that reminded senders of educational hours (March 2016, modified June 2016). Change in the interruption frequency was sustained over the following six months to the end of the observation period in December 2016.

Incoming phone call logs were available from April 2015 to December 2016, with a mean of $0.62(95 \% \mathrm{Cl}, 0.56$ to 0.67$)$ calls per team per educational hour, which did not change over the study period (Supplementary Figure 2). The overall number of calls to team smartphones also did not change during the measurement period. Incoming email data were available from October 2014 to December 2016, with a mean of 0.94 (95\% $\mathrm{Cl}, 0.88$ to 1.0$)$ emails per team per educational hour, which did not change over the study period (Supplementary Figure 3). Internal medicine service discharges, "Code Blue" announcements, and Critical Care Outreach Team consultations remained stable over the measurement period.

Independent ranking of the combined four-week samples of educational text interruptions from 2014 and 2016 revealed an initial three-way agreement on $257 / 455$ (56\%) messages (Fleiss Kappa 0.298, fair agreement), which increased to 405/455 (89\%) messages after the first joint assessment and reached full consensus after a third joint assessment that included classifying all messages that communicated institution-defined "critical lab" values as "urgent."

Overall, 71 (16\%) messages were classified as "urgent," 346 (76\%) as "nonurgent," and 38 (8\%) as "indeterminate." After unblinding of the message date and time, 273 text messages 
were received during the baseline measurement period (November 17 to December 14, 2014) and 182 messages were received during the equivalent time period two years later (November 14 to December 11, 2016), consistent with the reduced volume of educational interruptions observed (Figure 4). A total of 426 (94\%) messages were sent by nurses, and the remaining ones were sent by pharmacists $(n=20)$, ward clerks $(n=3)$, social workers $(n=4)$, speech language pathologist ( $=1)$, or device administrator $(n=1)$.

The proportion of "nonurgent" messages decreased from $223 / 273$ (82\%) in 2014 to $123 / 182$ (68\%) in 2016 ( $P \leq .01)$. Although the absolute number of urgent messages remained similar (33 in 2014 and 38 in 2016), the proportion of "urgent" messages increased from $12 \%$ to $21 \%$ of the total messages received ( $P=.02)$. Seventeen (6\%) messages had indeterminate frequency in 2014 compared to 21 (11.5\%) in 2016 (NS).

An audit of consecutive "FYI" messages (November 14-December 11,2016$)$ revealed an initial agreement in 384/431 (89\%), reaching full consensus after repeated joint assessments. A total of 406 (94\%) "FYI" messages were appropriately sent, while 10 (2\%) represented urgent communications that should have been sent as interruptions. In 15 (4\%) cases, the appropriateness of the message was indeterminate.

\section{DISCUSSION}

Sequential interventions over a 36-month period were associated with reduced nonurgent text message interruptions during educational hours. A clinical communication process was formally defined to accurately match message urgency with communication modality. A "noninterrupt" option allowed nonurgent text messages to be posted to an electronic message board, rather than causing real-time interruption, thereby reducing the overall volume of interrupting text messages. Modifying the interface to alert potential senders to protected educational hours was associated with reductions in educational interruptions. Through a blinded analysis of the text message content between 2014 and 2016, we determined that nonurgent educational interruptions were significantly reduced, and the number of urgent communications remained constant. Reduced nonurgent interruptions have the potential to improve the learning climate on the medical teaching unit during protected educational hours.

At baseline, $82 \%$ of the sampled text messages sent during educational hours across both sites were considered nonurgent. The estimated proportion of urgent messages varies in the literature $(5 \%-34 \%)^{13-18}$ possibly due to center-specific methods of defining and measuring urgent messages. For example, different assessor training backgrounds, different numbers of assessors, and varying institutional policies are described. ${ }^{13-17}$ We considered an urgent message to require a response or action within one hour or to represent an established "critical lab value" as per the institution. The high proportion of nonurgent interruptions found in this study and other works demonstrates the widespread nature of this problem within inpatient hospital settings; this phenomenon could potentially lead to unintended consequences on efficiency and medical education.
Few other initiatives have aimed to reduce interruptions to medical trainees during educational sessions. At one center, replacing numeric pagers with alphanumeric pagers decreased the need to return pages during educational sessions but did not decrease the overall number of pages. ${ }^{21}$ Another center implemented an inbox tool that reduced daytime nonurgent numeric pages. ${ }^{15}$ Similar to our center's previous experience, ${ }^{11}$ the total number of communications increased with the creation of the inbox tool. ${ }^{15}$ Unexpectedly, the introduction of an "FYI" option for senders in March 2015 did not increase the total number of messages.

Increasing use of text messages for communication between physicians and allied health professions has resulted in higher volumes of interruptions compared with conventional paging. ${ }^{6,79}$ Excessive interruptions create a "crisis mode" work climate, ${ }^{10}$ which could compromise patient safety ${ }^{25-27}$ and hamper trainees' attainment of educational objectives. ${ }^{18-20,23}$ During educational sessions, audible text, phone call, and email interruptions disrupt all learners in addition to the resident receiving the message. The creation of the "FYI" message option in March 2015 was associated with reduced overall daily interruptions, which may improve efficiency in residents' clinical duties ${ }^{17,18}$ and minimize multi-tasking that could lead to errors. ${ }^{28}$ However, adding a real-time notification during educational hours (March 2016, modified June 2016) exerted the greatest impact specifically on educational interruptions. Engaging physicians in the creation and ongoing modification of instant-messaging interfaces can help customize technology to meet the needs of users. ${ }^{15,29}$ Our work provides a strategy for improving communication between nurses and physicians in a teaching hospital setting, by achieving consensus on levels of urgency of different messages, providing a non-interrupting message option, and providing nurses with real-time information about educational hours.

Potential unintended consequences of the interventions require consideration. Discouraging interruptions may have reduced urgent patient care communications but were mitigated by enabling senders to ignore/override interruption warnings. We did not observe an increase in the number of overall calls to team devices, "Code Blues," or critical care team consultations. However, we found that a very small (2\%) but important group of "FYI" messages should have been sent as urgent interrupting messages, thereby underscoring the necessity for continuous feedback to senders on the clinical communication process.

Our study has limitations. Although educational interruptions can cause fragmented learning at our institution, ${ }^{19}$ the impact of reduced interruptions on the quality of educational sessions can only be inferred because we did not formally assess resident or staff physician perceptions on this outcome during the interventions. Moreover, we were unable to quantify interruptions received through personal smartphones, a frequent method of physician-physician communication. ${ }^{30}$ Phone calls are the most intrusive of interruptions but were not the focus of interventions. Future work must consider documenting perceived appropriateness of calls in real time, similar to 
previous studies assessing paging urgency. ${ }^{13,14,18}$ Biased ranking of message urgency was minimized by utilizing three independent adjudicators blinded to message date throughout the adjudication process and by applying established communication guidelines where available. Nevertheless, retrospective assessment of message urgency could be limited by a lack of clinical context, which may have been more apparent to the original sender and the recipient. Finally, at our center, a close relationship with the communication platform programmer made sequential modifications possible, while other institutions may have limited ability to make such changes. A different approach may be useful in some cases, such as modifying academic teaching times to limit interruptions. ${ }^{23}$

In a large academic center, a high number of interrupting smartphone messages cause unnecessary distractions and reduce learning during educational hours. "Nonurgent" educational interruptions were reduced through successive improvement cycles, and ultimately by modifying the program interface to alert senders of educational hours. Further reduction in interruptions and sustainability may be achieved by studying phone call interruptions and by formalizing audit and feedback of sender's adherence to standardized clinical communication methods.

\section{Acknowledgments:}

Dr. Wu is supported by an award from the Mak Pak Chiu and Mak-Soo Lai Hing Chair in General Internal Medicine, University of Toronto. The authors would like to acknowledge Jason Uppal for his ongoing contribution to the improvement of clinical text message communications at our institution.

Disclosures: The authors have nothing to disclose.

\section{References:}

1. Wu R, Lo V, Morra D, et al. A smartphone-enabled communication system to improve hospital communication: usage and perceptions of medical trainees and nurses on general internal medicine wards. J Hosp Med. 2015;10(2):83-89.

2. Smith CN, Quan SD, Morra D, et al. Understanding interprofessional communication: a content analysis of email communications between doctors and nurses. App/ Clin Inform. 2012;3(1):38-51.

3. Frizzell JD, Ahmed B. Text messaging versus paging: new technology for the next generation. J Am Coll Cardiol. 2014;64(24):2703-2705.

4. Wu RC, Morra D, Quan S, et al. The use of smartphones for clinical communication on internal medicine wards. J Hosp Med. 2010;5(9):553-559.

5. Ighani F, Kapoor KG, Gibran SK, et al. A comparison of two-way text versus conventional paging systems in an academic ophthalmology department. J Med Syst. 2010;34(4):677-684.

6. Wu R, Rossos P, Quan S, et al. An evaluation of the use of smartphones to communicate between clinicians: a mixed-methods study. J Med Internet Res. 2011;13(3):e59.

7. Wu RC, Lo V, Morra D, et al. The intended and unintended consequences of communication systems on general internal medicine inpatient care delivery: a prospective observational case study of five teaching hospitals. J Am Med Inform Assoc. 2013;20(4):766-777.

8. Patel N, Siegler JE, Stromberg N, Ravitz N, Hanson CW. Perfect storm of inpatient communication needs and an innovative solution utilizing smartphones and secured messaging. App/ Clin Inform. 2016;7(3):777-789.

9. Aungst TD, Belliveau P. Leveraging mobile smart devices to improve interprofessional communications in inpatient practice setting: A literature review. J Interprof Care. 2015;29(6):570-578.

10. Vaisman A, Wu RC. Analysis of Smartphone Interruptions on Academic General Internal Medicine Wards. Frequent Interruptions may cause a 'Crisis Mode' Work Climate. Appl Clin Inform. 2017;8(1):1-11.

11. Quan SD, Wu RC, Rossos PG, et al. It's not about pager replacement: an indepth look at the interprofessional nature of communication in healthcare. J Hosp Med. 2013;8(3):137-143.

12. Quan SD, Morra D, Lau FY, et al. Perceptions of urgency: defining the gap between what physicians and nurses perceive to be an urgent issue. Int J Med Inform. 2013;82(5):378-386.

13. Katz MH, Schroeder SA. The sounds of the hospital. Paging patterns in three teaching hospitals. N Engl J Med. 1988;319(24):1585-1589.

14. Patel R, Reilly K, Old A, Naden G, Child S. Appropriate use of pagers in a New Zealand tertiary hospital. N Z Med J. 2006;119(1231):U1912.

15. Ferguson A, Aaronson B, Anuradhika A. Inbox messaging: an effective tool for minimizing non-urgent paging related interruptions in hospital medicine provider workflow. BMJ Qual Improv Rep. 2016;5(1):u215856.w7316.

16. Luxenberg A, Chan B, Khanna R, Sarkar U. Efficiency and interpretability of text paging communication for medical inpatients: A mixed-methods analysis. JAMA Intern Med. 2017;177(8):1218-1220.

17. Ly T, Korb-Wells CS, Sumpton D, Russo RR, Barnsley L. Nature and impact of interruptions on clinical workflow of medical residents in the inpatient setting. J Grad Med Educ. 2013;5(2):232-237.

18. Blum NJ, Lieu TA. Interrupted care. The effects of paging on pediatric resident activities. Am J Dis Child. 1992;146(7):806-808.

19. Wu RC, Tzanetos K, Morra D, Quan S, Lo V, Wong BM. Educational impact of using smartphones for clinical communication on general medicine: more global, less local. J Hosp Med. 2013;8(7):365-372.

20. Katz-Sidlow RJ, Ludwig A, Miller S, Sidlow R. Smartphone use during inpatient attending rounds: prevalence, patterns and potential for distraction. J Hosp Med. 2012;7(8):595-599.

21. Wong BM, Quan S, Shadowitz S, Etchells E. Implementation and evaluation of an alpha-numeric paging system on a resident inpatient teaching service. J Hosp Med. 2009;4(8):E34-E40.

22. Conard MA MR. Interest level improves learning but does not moderate the effects of interruptions: An experiment using simultaneous multitasking. Learn Individ Differ. 2014;30:112-117

23. Zastoupil L, Mclntosh A, Sopfe J, et al. Positive impact of transition from noon conference to academic half day in a pediatric residency program. Acad Pediatr. 2017;17(4):436-442.

24. Lo V, Wu RC, Morra D, Lee L, Reeves S. The use of smartphones in general and internal medicine units: a boon or a bane to the promotion of interprofessional collaboration? J Interprof Care. 2012;26(4):276-282.

25. Patterson ME, Bogart MS, Starr KR. Associations between perceived crisis mode work climate and poor information exchange within hospitals. J Hosp Med. 2015;10(3):152-159.

26. Laxmisan A, Hakimzada F, Sayan OR, Green RA, Zhang J, Patel VL. The multitasking clinician: decision-making and cognitive demand during and after team handoffs in emergency care. Int J Med Inform. 2007;76(11-12):801-811.

27. Westbrook JI, Woods A, Rob MI, Dunsmuir WT, Day RO. Association of interruptions with an increased risk and severity of medication administration errors. Arch Intern Med. 2010;170(8):683-690.

28. Collins S, Currie L, Patel V, Bakken S, Cimino JJ. Multitasking by clinicians in the context of CPOE and CIS use. Stud Health Technol Inform. 2007;129(Pt 2):958-962.

29. Huang ME. It is from mars and physicians from venus: Bridging the gap. PM R. 2017;9(5S):S19-S25

30. Tran K, Morra D, Lo V, Quan S, Wu R. The use of smartphones on General Internal Medicine wards: A mixed methods study. Appl Clin Inform. 2014;5(3):814-823. 